\title{
Frequency Swept Fiber Ring Laser Based on Optical Parametric Process With Single-Longitudinal-Mode Operation
}

\author{
Kim K. Y. Cheung, Sigang Yang, Yue Zhou, and Kenneth K. Y. Wong, Senior Member, IEEE
}

\begin{abstract}
A stable and wavelength-tunable single-longitudinalmode (SLM) oscillation with sweeping rate of $1.17 \mathrm{kHz}$ based on a fiber-optical parametric oscillator (FOPO) is proposed and demonstrated. In order to perform sweeping SLM operations, three types of filters, a fiber Fabry-Pérot tunable filter (FFP-TF), a subring cavity, and an autotracking filter which is formed by an unpumped erbium-doped fiber (EDF), are incorporated inside the cavity. The output of the laser can be swept continuously from 1530 to $1543 \mathrm{~nm}$ and 1569 to $1584 \mathrm{~nm}$ with the average output power exceeding $3 \mathrm{dBm}$. The short-term linewidth of the laser output measured by self-homodyne method is about $66.6 \mathrm{kHz}$ with a side-mode suppression ratio of $60 \mathrm{~dB}$ at $1538 \mathrm{~nm}$.
\end{abstract}

Index Terms-Fiber loop mirror, fiber-optical parametric oscillator, fiber ring laser, single-longitudinal mode (SLM).

\section{INTRODUCTION}

$\mathbf{S}$ INGLE-LONGITUDINAL-MODE lasers are attractive in myriad applications, ranging from optical communications, sensing and spectroscopy, which have been studied extensively. For example, it has been demonstrated that SLM laser can act as an amplitude modulation (AM) transmitter source in video transmission [1] and wavelength-division multiplexing (WDM) communication systems. SLM lasers can also be used for gas spectroscopy by tuning the narrow linewidth laser across a gas absorption line [2]-[4]. Single-frequency operations based on EDF acting as a gain medium have been demonstrated before [5], [6]. For the EDF, the amplification window is limited by the material properties hence the lasing wavelength is limited. With the high gain [7] and wide gain bandwidth [8] provided by fiber optical parametric amplifier (FOPA), the flexibility of tuning its parametric oscillating wavelength significantly increased [9].

Parametric amplification and oscillation can be obtained by either $\chi^{(2)}[10]$ or $\chi^{(3)}[8]$ nonlinearity. For the OPO that use $\chi^{(2)}$ nonlinear crystal, the cavity length is relatively short and hence the longitudinal mode spacing is large. SLM lasing can

Manuscript received May 18, 2010; revised October 28, 2010; accepted November 20, 2010. Date of publication December 03, 2010; date of current version January 21,2011 . This work was supported in part by grants from the Research Grants Council of the Hong Kong Special Administrative Region, China (Projects HKU 7179/08E and HKU 7183/09E).

The authors are with the Department of Electrical and Electronic Engineering, the University of Hong Kong, Hong Kong (e-mail: cheungky@eee. hku.hk; yangsg08@gmail.com; sgyang@eee.hku.hk; yzhou@eee.hku.hk; kywong@eee.hku.hk).

Color versions of one or more of the figures in this letter are available online at http://ieeexplore.ieee.org.

Digital Object Identifier 10.1109/LPT.2010.2096464

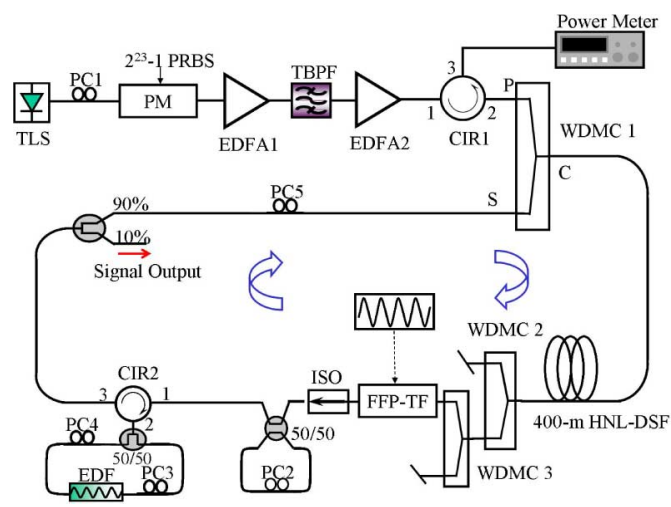

Fig. 1. Configuration of the sweeping SLM FOPO.

be easily obtained by inserting a traditional bandpass filter inside the cavity. For the fiber-integrated OPO, long fiber is commonly deployed in order to obtain enough gain to compensate the cavity loss; especially for the case with continuous-wave $(\mathrm{CW})$ pump. The longitudinal mode spacing $(\delta v)$ can be calculated by $\delta v=c / n L$ where $c$ is the speed of light in vacuum, $n$ is the refractive index of the ring and $L$ is the cavity length. As the longitudinal mode spacing is inversely proportional to the total cavity length, and with the cavity length in the order of hundred meters, the longitudinal modes spacing will be in the order of megahertz or kilohertz [11]. Such narrow mode spacing is extremely difficult to be filtered by an optical filter and mode hopping will occur easily.

In this Letter, an unpumped EDF and a subring cavity are incorporated inside the main cavity of FOPO in order to establish linewidth narrowing and single-mode operation [1], [11]. The experiment is divided into two parts; the first part is to verify the SLM laser operating at fixed wavelength while the second part is to investigate the sweeping SLM laser. To provide sweeping, a fiber Fabry-Pérot tunable bandpass filter (FFP-TF) is inserted inside the cavity. The FFP-TF determines the oscillating wavelength by applying the sinusoidal electrical signal to the built-in piezoelectric transducer (PZT) in FFP-TP. The fastest sweeping frequency we demonstrated is $1.17 \mathrm{kHz}$ with $15-\mathrm{nm}$ sweeping bandwidth.

\section{EXPERIMENTAL SETUP}

The proposed configuration of the sweeping SLM FOPO is shown in Fig. 1. A CW pump with a wavelength of $1555.5 \mathrm{~nm}$ was produced by a tunable laser source (TLS). It was then phase modulated by a phase modulator (PM) with $10 \mathrm{~Gb} / \mathrm{s} 2^{23}-1$ pseudorandom bit sequence (PRBS) in order to suppress the 
stimulated Brillouin scattering (SBS) effect [12]. The polarization controller ( $\mathrm{PC} 1)$ was used to align the state of polarization (SOP) of the pump to the transmission axis of the PM. The dithered pump was then amplified by a two-stage erbium-doped amplifier (EDFA1 and EDFA2) with a tunable bandpass filter (TBPF) inserted between to remove the amplified spontaneous emission (ASE) noise. A circulator (CIR1) was used to prevent the reflected pump power and observe any significant SBS.

The input pump power was $28.78 \mathrm{dBm}$ and was then coupled into a spool of 400-m highly-nonlinear dispersion-shifted fiber (HNL-DSF) with zero-dispersion wavelength at $1554 \mathrm{~nm}$ via the P-port (transmission band: 1554.89-1563.89 nm) of a WDM coupler (WDMC1). The HNL-DSF had nonlinear coefficient of $14 \mathrm{~W}^{-1} \mathrm{~km}^{-1}$, a loss of $\sim 1.5 \mathrm{~dB}$ and a dispersion slope of $0.02 \mathrm{ps} / \mathrm{nm}^{2} / \mathrm{km}$. To prevent high pump power from damaging to the FFP-TF, WDMC2 and WDMC3 were used to remove the residual pump. A sinusoidal electrical wave at frequency $1.17 \mathrm{kHz}$ was applied to FFP-TF so that the lasing wavelength was sweeping at $1.17 \mathrm{kHz}$. The FFP-TF used in this experiment had a free spectral range (FSR) of $160 \mathrm{~nm}$ at $1550 \mathrm{~nm}$ and a finesse of 750. An isolator (ISO) in a cavity was used to ensure unidirectional operation. In order to increase the longitudinal mode spacing, a subring cavity filter formed by a 50/50 coupler and PC2 was added after the ISO. The length of the main cavity was $428 \mathrm{~m}$ while the length of the subring cavity was $3.1 \mathrm{~m}$. Insertion of subring cavity could increase the FSR from $478 \mathrm{kHz}$ to $66 \mathrm{MHz}$ thus it will be easier to obtain the SLM operation. Furthermore, a 3-m unpumped EDF, acting as a saturable absorber, was coupled to the main cavity by CIR2 which was also acted as an isolator. In the unpumped EDF, two counter-propagating waves form a standing wave and produce a periodic spatial hole burning (SHB) [13] thus enables SLM operation in combination with the subring cavity. Since the operating wavelength of the unpumped EDF auto-tracks with the applied wavelength, it can be incorporated with the FFP-TF to obtain sweeping SLM laser. The two in-line PCs (PC3 and PC4) were used to ensure that the SOP of the light was properly controlled while a 90/10 optical coupler in the main cavity provided $90 \%$ feedback signal and $10 \%$ output. $90 \%$ feedback was then combined with the pump through S-port (transmission band: $1500-1551.39 \mathrm{~nm}, 1567.39-1600 \mathrm{~nm}$ ) of WDMC1. PC5 was used to align the signal's SOP with the pump so as to maximize the signal gain. The output optical spectra were measured by optical spectrum analyzer (OSA) while the radio frequency (RF) spectra were measured by self-homodyne method using electrical spectrum analyzer (ESA).

\section{EXPERIMENTAL RESULTS AND DISCUSSION}

\section{A. Fixed Wavelength SLM Laser}

The first part of the experiment is to verify the performances of the SLM laser operating at fixed wavelength. A constant DC voltage instead of a sinusoidal electrical signal was applied to the FFP-TF hence we could adjust the lasing wavelength manually. Fig. 2 shows the single frequency laser spectra measured by OSA with optical resolution of $0.06 \mathrm{~nm}$. The laser was tuned from 1524 to $1541 \mathrm{~nm}$ and 1567 to $1582 \mathrm{~nm}$ with average output power of $4.1 \mathrm{dBm}$. The discontinuity of the spectra

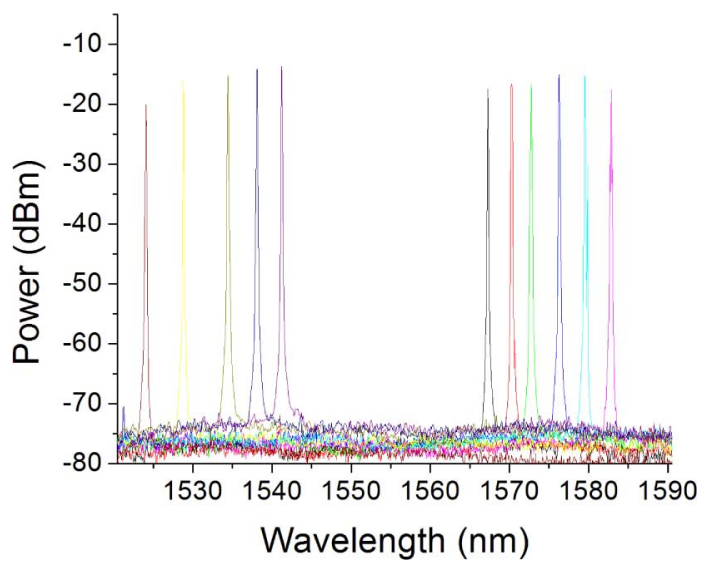

Fig. 2. Single frequency laser spectra.

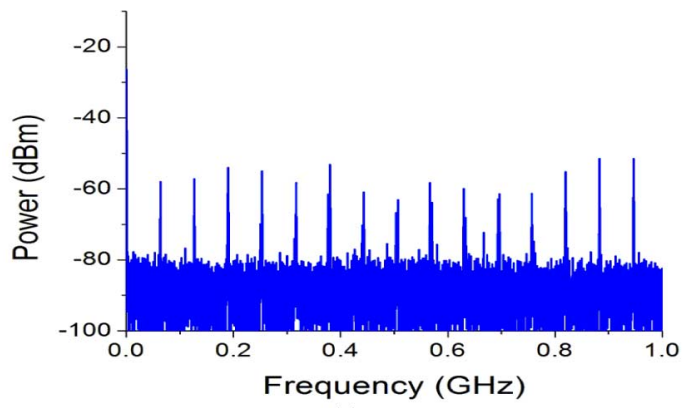

(a)

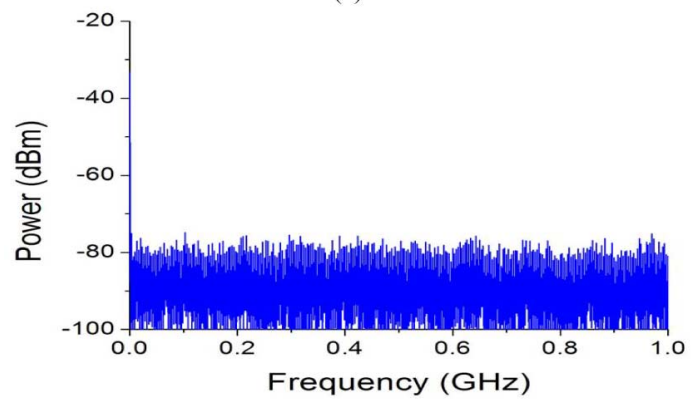

(b)

Fig. 3. Measured self-homodyne spectra for the lasing wavelength at $1575 \mathrm{~nm}$ : (a) With subring cavity while EDF loop is removed. (b) With subring cavity and EDF loop connected.

was due to the WDMC used to filter the pump and the characteristic gain spectrum of a typical one-pump FOPA. The shortterm linewidth of the laser output measured by self-homodyne method was about $66.6 \mathrm{kHz}$ with side-mode suppression ratio of $60 \mathrm{~dB}$ measured at $1538 \mathrm{~nm}$.

Fig. 3(a) shows the beating signal spectrum measured by the ESA at the $10 \%$ output port for the case that subring cavity was present while the EDF loop was removed. The operating wavelength was $1575 \mathrm{~nm}$ with beating signal at $66 \mathrm{MHz}$. Fig. 3(b) shows the beating signal spectrum when the EDF loop was inserted. From the spectra, it can be observed the unpumped EDF greatly suppressed the side modes and enabled SLM operation.

\section{B. Sweeping SLM Laser}

Second part of the experiment investigated the sweeping operation of the proposed SLM laser. Fig. 4 shows the sweeping 


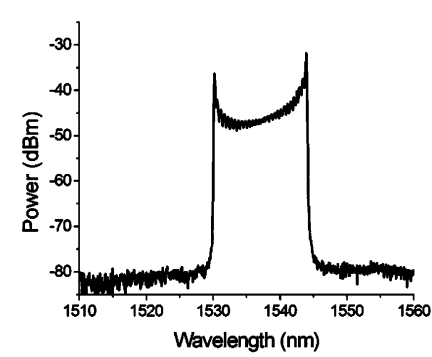

(a)

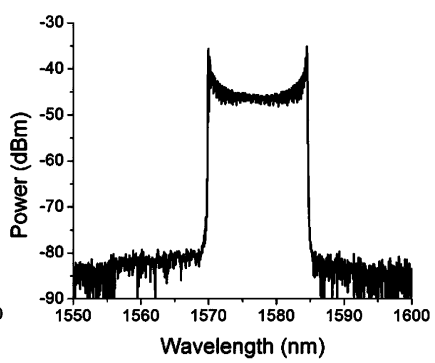

(b)
Fig. 4. Sweeping laser spectra.

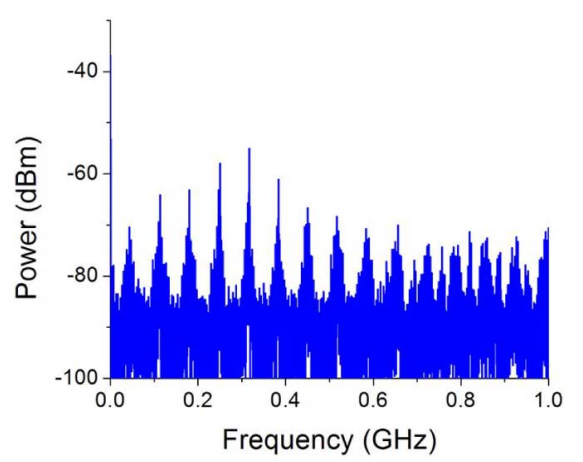

(a)

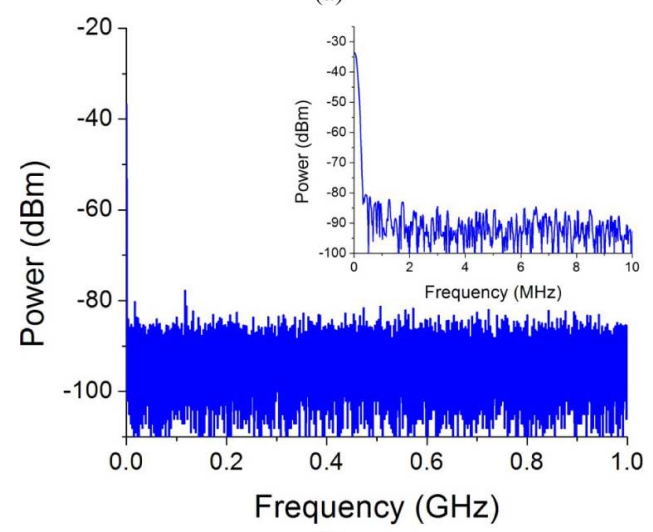

(b)

Fig. 5. Measured self-homodyne spectra for sweeping from 1569 to $1584 \mathrm{~nm}$ : (a) With subring cavity while EDF loop was removed. (b) With subring cavity and EDF loop connected and the inset is the zoom-in version.

spectra with average output power exceeds $3 \mathrm{dBm}$ at a sweeping rate of $1.17 \mathrm{kHz}$.

Fig. 4(a) shows the sweeping spectrum from 1530 to $1543 \mathrm{~nm}$ while Fig. 4(b) shows the corresponding spectrum from 1569 to $1584 \mathrm{~nm}$. The tunable range was limited by the gain bandwidth of the FOPA. Fig. 5 shows the RF spectrum when the laser is sweeping from 1569 to $1584 \mathrm{~nm}$. Fig. 5(a) shows that there exist noisy and unstable spikes which indicated multimode operation. When comparing Fig. 5(a) with Fig. 3(a), it can be observed that the beating signals in Fig. 5(a) show broader spectra than that in Fig. 3(a) which is due to the sweeping operation. Fig. 5(b) shows the RF spectrum in which the subring cavity and EDF were connected to the main cavity. The inset in Fig. 5(b) is the zoomed-in version of Fig. 5(b) with stopping frequency at 10 MHz. In Fig. 5(b), only direct current peak can be observed, but not other signal. It indicates that the EDF loop and the sub- ring cavity can suppress the fundamental main cavity frequency and enable SLM operations. Similar RF spectrum could be obtained when the laser swept from $1530 \mathrm{~nm}$ to $1543 \mathrm{~nm}$.

\section{CONCLUSION}

We have demonstrated a novel stable and wavelength-tunable SLM fiber laser based on FOPO with sweeping rate of $1.17 \mathrm{kHz}$. The sweeping bandwidth was from 1530 to $1543 \mathrm{~nm}$ and 1569 to $1584 \mathrm{~nm}$ with the average power exceeded $3 \mathrm{dBm}$. The short-term linewidth of the laser output was about $66.6 \mathrm{kHz}$ with side-mode suppression ratio of $60 \mathrm{~dB}$ at $1538 \mathrm{~nm}$. The SLM operation is done by an unpumped EDF and a subring cavity. The sweeping bandwidth can be further increased by increasing the pump power used.

\section{ACKNOWLEDGMENT}

The authors would like to acknowledge Sumitomo Electric Industries for providing HNL-DSF.

\section{REFERENCES}

[1] C. C. Lee, Y. K. Chen, and S. K. Liaw, "Single-longitudinal-mode fiber laser with a passive multiple-ring cavity and its application for video transmission," Opt. Lett., vol. 23, pp. 358-360, Mar. 1998.

[2] S. K. Kim, G. Stewart, W. Johnstome, and B. Culshaw, "Modehop-free single-longitudinal-mode erbium-doped fiber laser frequency scanned with a fiber ring resonator," Appl. Opt., vol. 38, pp. 5154-5157, Aug. 1999.

[3] T. Liu, Y. H. Wang, R. Dumke, A. Stejskal, Y. N. Zhao, J.Zhang, Z. H. Lu, L. J. Wang, T. Becker, and H. Walther, "Narrow linewidth light soruce for an ultraviolet optical frequency standard," Appl. Phys. B., vol. 87, pp. 227-232, Mar. 2007.

[4] A. Y. Nevsky, U. Bressel, I. Ernsting, C. Eisels, M. Okhapkin, S Schiller, A. Gubenko, D. Livshits, S. Mikhrin, I. Krestnikov, and A. Kovsh, "A narrow-line-width externalcavity quantum dot laser for high-resolution spectroscopy in the near-infrared and yellow spectral ranges," Appl. Phys. B, vol. 92, pp. 501-507, May 2008.

[5] G. J. Cowle, D. N. Payne, and D. Reid, "Single-frequency travelling-wave erbium-doped fiber loop laser,' Electron. Lett., vol. 27, pp. 229-230, Jan. 1991.

[6] Y. Cheng, J. T. Kringlebotn, W. H. Loh, R. I. Laming, and D. N. Payne, "Stable single-frequency travelling-wave fiber loop laser with intergral saturable-absorber-based tracking narrow-band filter," Opt. Lett., vol. 20, pp. 875-877, Apr. 1995.

[7] T. Torounidis, P. A. Andrekson, and B. E. Olsson, "Fiber-optical parametric amplifier with 70-dB gain," IEEE Photon. Technol. Lett., vol. 18, no. 10, pp. 1194-1196, May 15, 2006.

[8] M. E. Marhic, K. K. Y. Wong, and L. G. Kazovsky, "Wide-band tuning of the gain spectrum of one pump optical parametric amplifiers," IEEE J. Sel. Topics Quantum Electron., vol. 10, no. 5, pp. 1133-1141, Sep./ Oct. 2004.

[9] Y. Zhou, K. K. Y. Cheung, S. Yang, and K. K. Y. Wong, "Widely tunable picosecond optical parametric oscillator using highly nonlinear fiber,' Opt. Lett., vol. 34, pp. 989-991, Apr. 2009.

[10] D. J. M. Stothard, I. D. Lindsay, and M. H. Dunn, "Continuous-wave pump-enhanced optical parametric oscillator with ring resonator for wide and continuous tuning of single-frequency radiation," Opt. Express, vol. 12, pp. 502-511, Feb. 2004.

[11] S. Yang, K. K. Y. Cheung, Y. Zhou, and K. K. Y. Wong, "Tunable single-longitudinal-mode fiber optical parametric oscillator," Opt. Lett., vol. 35, pp. 481-483, Feb. 2010.

[12] S. K. Korotky, P. B. Hansen, L. Eskildsen, and J. J. Veselka, "Efficient phase modulation shceme for suppressing stimulated Brillouin scattering," in Proc. Tech. Dig. Int. Conf. Integrated Optics Optical Fiber Communications, Hong Kong, 1995, vol. 2, pp. 110-111, Paper WD2-1.

[13] M. Mastuura and N. Kishi, "Frequency control characteristics of a single-frequency fiber laser with an external light injection," IEEE J. Sel. Topics Quantum Electron., vol. 7, no. 1, pp. 55-58, Jan./Feb. 2001. 